\title{
Synthesis of nanosized nickel ferrites by shock waves and their magnetic properties
}

\author{
Jianjun Liu ${ }^{\mathrm{a} *}$, Hongliang $\mathrm{He}^{\mathrm{b}}$, Xiaogang $\mathrm{Jin}^{\mathrm{b}}$, Zhengping $\mathrm{Hao}^{\mathrm{c}}$, \\ Zhuangqi $\mathrm{Hu}^{\mathrm{d}}$ \\ ${ }^{a}$ Faculty of Sciences, Beijing University of Chemical Technology, Beijing, 100029, P.R. of China \\ ${ }^{\mathrm{b}}$ Laboratory for Shock Wave and Detonation Physics Research, Southwest Institute of Fluid Physics, \\ P.O. Box 919, Mianyang, 621900, P.R. of China \\ ${ }^{\mathrm{c}}$ Research Center for Eco-Environmental Sciences, Chinese Academy of Sciences, Beijing, 100085, \\ P.R. of China \\ ${ }^{\mathrm{d}}$ Shenyang National Laboratory for Materials Science, Shenyang, 110016, P.R. of China \\ (Refereed) \\ Received 17 January 2001; accepted 9 May 2001
}

\begin{abstract}
The nanosized mixture powders of ferric oxide and nickel oxide prepared by the coprecipitation in a stoichiometric ratio to form $\mathrm{NiFe}_{2} \mathrm{O}_{4}$ were subjected to shock wave treatment. The nanosized nickel ferrites with different particle sizes were synthesized by means of varying initial packing density of the mixture. Compared with nickel ferrite prepared by conventional calcination, the magnetization of ones by shock wave treatment increases significantly. It is believed that shock wave treatment is a novel method for the preparation of nanosized powders of composite metal oxides with particular physical properties. (C) 2001 Elsevier Science Ltd. All rights reserved.
\end{abstract}

Keywords: A. High pressure; B. Nanoparticle; C. Magnetic properties

\section{Introduction}

The shock-induced chemical reactions leading to synthesis of compounds in powder mixtures occurred under conditions of the microsecond-scale duration of the high pressure, stress, strain-

* Corresponding author.

E-mail address: liujianjun717@163.net (J. Liu). 
rate, and temperature states. Such high-rate chemical reactions can be advantageously utilized to synthesize materials with novel phases and unique microstructures, or to generate radically modified materials with physically interesting or technologically useful properties [1-3]. Many works concerning the synthesis and modification of complex metal oxides by shock waves have been published [4,5], but only a few deal with the modification of nickel ferrites purchased, and these works focus on the properties of the magnetism and microwave absorption of nickel ferrites [6,7]. No investigation on the synthesis of nickel ferrites, especially nanosized nickel ferrites by shock wave treatment and the magnetic properties has been published.

In this article, we report the preliminary results of our study on the synthesis of nanosized nickel ferrites and their magnetic properties by shock wave treatment, and discuss possible reasons for the formation of nanosized nickel ferrites and the increase of their magnetizations.

\section{Experimental}

The precursor of samples $\left(\mathrm{NiO}+\mathrm{Fe}_{2} \mathrm{O}_{3}\right.$ mixture) was prepared by the coprecipitation by adding ammonia water to the aqueous solution of nickel nitrate and ferric nitrate in a molar ratio of 1:2. The precipitate was filtered, washed, and dried at $120^{\circ} \mathrm{C}$ for $10 \mathrm{~h}$, and then ground and sieved (100 mesh). The product was a nanosized mixture of ferric oxide and nickel oxide that is used as a precursor for subsequent treatments.

The detonation of a $\phi 100 \times 150-\mathrm{mm}$ nitromethane explosive charge was used to accelerate a steel plate to $2.63 \mathrm{~km} / \mathrm{s}$, to impact the steel recovery device and produce strong shock waves. The initial shock wave pressure in the samples calculated by a simple one-dimensional hydrodynamic method with two packing densities $\left(\rho_{1}=2.86 \mathrm{~g} / \mathrm{cm}^{3}\right.$ and $\rho_{2}=2.23 \mathrm{~g} / \mathrm{cm}^{3}$, corresponding to 53.2 and $41.4 \%$ of the crystallite density of $\mathrm{NiFe}_{2} \mathrm{O}_{4}$, respectively) was 28 and $23 \mathrm{GPa}$, respectively. Details of the shock wave loading recovery devices can be found elsewhere [8]. Unlike the synthesis by shock wave, conventional nickel ferrite of normal spinel was synthesized by calcination of the precursor at $700^{\circ} \mathrm{C}$ for $10 \mathrm{~h}$.

XRD patterns were obtained on a Rigaku D/MAX-RA X-ray diffractometer. TEM images and electron diffraction patterns were taken on a JEM-100CX electron microscope. Diameter of the electron beam was $1.0 \mu \mathrm{m}$. The measurement of the magnetization carried out on a vibration magnetometer of CF-1(made by Sino-France) with applied magnetic field of 0-65 kOe.

\section{Results and discussion}

\subsection{XRD patterns and TEM images}

The XRD patterns of samples are shown in Fig. 1. Fig. 1a is the XRD pattern of NF-3(by calcinations). The sharp peaks of nickel ferrite [9] (denoted by $*$ ) and weak peaks of $\alpha-\mathrm{Fe}_{2} \mathrm{O}_{3}$ (denoted by $\mathbf{~}$ ) indicate that high temperature calcination produces perfect crystalline spinel of the nickel ferrite. Fig. 1b and 1c are the XRD patterns of samples synthesized by shock wave (NF-2 and NF-1) with different packing densities. In contrast to Fig. 1a, the main peaks can be attributed to nickel ferrite, but XRD patterns of both NF-2 and NF-1, especially NF-1, 


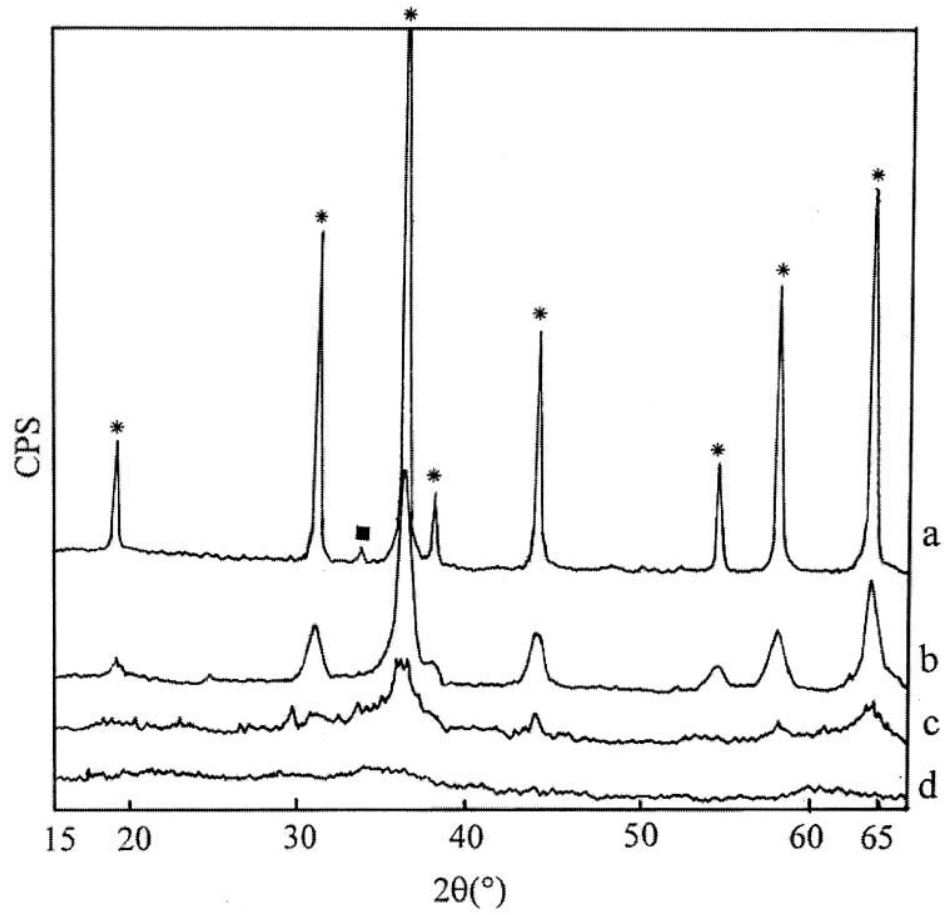

Fig. 1. XRD patterns of nickel ferrites synthesized by different methods and the precursor: (a) calcination (NF-3); $(b, c)$ shock wave loading with packing densities of 2.23 and $2.86 \mathrm{~g} / \mathrm{cm}^{3}$, respectively (NF-2 and NF-1); (d) the precursor prepared by coprecipitation ( NF-0).(* spinel nickel ferrite, $\mathbf{\square} \alpha-\mathrm{Fe}_{2} \mathrm{O}_{3}$ ).

show broadened line profiles resulting from reduction in crystallite size and residual lattice strain. In the XRD pattern of precursor (Fig. 1d), no diffraction peaks can be found.

In Fig. 2, the TEM images (a, b, c, and d) and the electron diffraction patterns (a-1 and b-1) of samples No. NF-0, NF-1, NF-2, and NF-3 are shown. The samples synthesized by calcination consist of particles with diameters about $100 \mathrm{~nm}$ (Fig. 2a). In the pattern of electron diffraction (Fig. 2a-1) there are distinct diffractions spots with some very weak diffraction rings. Fig. 2b and $2 \mathrm{~b}-1$ shows the corresponding results for the sample obtained by shock wave treatment with packing density of $\rho_{1}=2.86 \mathrm{~g} / \mathrm{cm}^{3}$ (No. NF-1). The particle size is much smaller (less than 10 $\mathrm{nm}$ ), and in the electron diffraction pattern (Fig. 2b-1) there are distinct diffraction rings. From the electron diffraction patterns, the distances between the lattice planes (d values) can be calculated (see Table 1); their values are identical to the standard values of nickel ferrite reported in ref. [9]. With consideration of Fig. 2 and Table 1, together with the XRD pattern (Fig. 1), it can be concluded that the main composition of the sample obtained by shock wave treatment is also spinel nickel ferrite; but it has very small nanosized particles and high densities of defects and dislocations; thus, it cannot give characteristic XRD patterns of spinel nickel ferrite. Fig. 2c shows the results for the sample obtained by shock wave treatments with packing density of $\rho_{2}=$ $2.23 \mathrm{~g} / \mathrm{cm}^{3}$ (NF-2). It can be seen that the particle size is about $30 \mathrm{~nm}$, which is larger than that of NF-1. In general, at about the same pressure of the shock wave, if only the adiabatic temperature rise in a porous material is considered, the lower the packing density, the higher the 


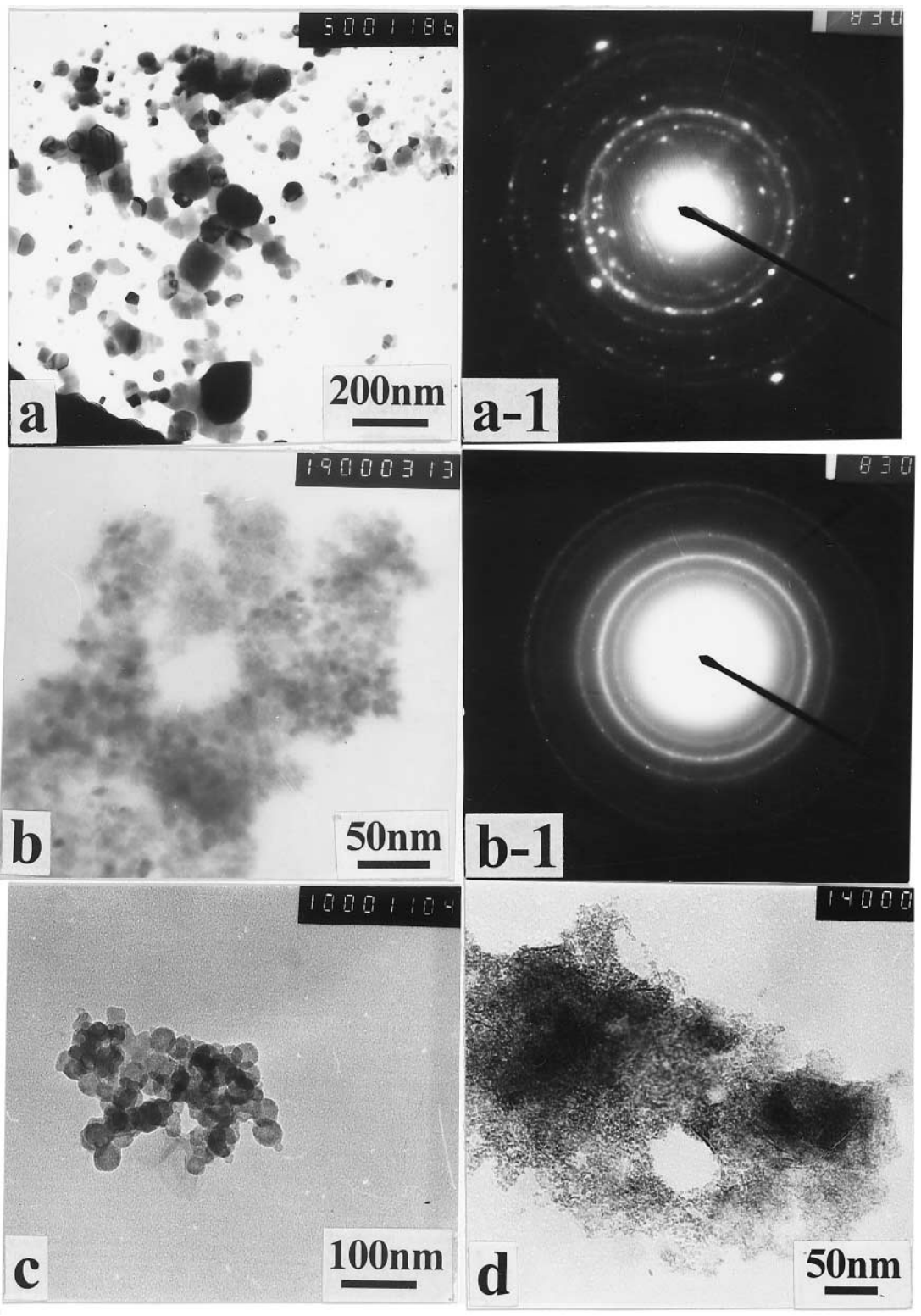

Fig. 2. TEM images (a, b, c, and d) and electron diffraction patterns (a-1 and b-1) of nickel ferrites synthesized by different methods and the precursor: (a,a-1) by calcination (NF-3); (b,b-1) by shock wave with packing density of $2.86 \mathrm{~g} / \mathrm{cm}^{3}$ (NF-1); (c) by shock wave with packing density of $2.23 \mathrm{~g} / \mathrm{cm}^{3}$ (NF-2); (d) the precursor prepared by coprecipitation (NF-0).

shock temperature [10]. Hence, a shock wave can produce higher shock temperature in the sample (NF-2) of lower packing density, which leads to the formation of larger perfect crystallites. The TEM of precursor (Fig. 2d) shows that it has very small particle sizes, ranging from 5 to $10 \mathrm{~nm}$. 


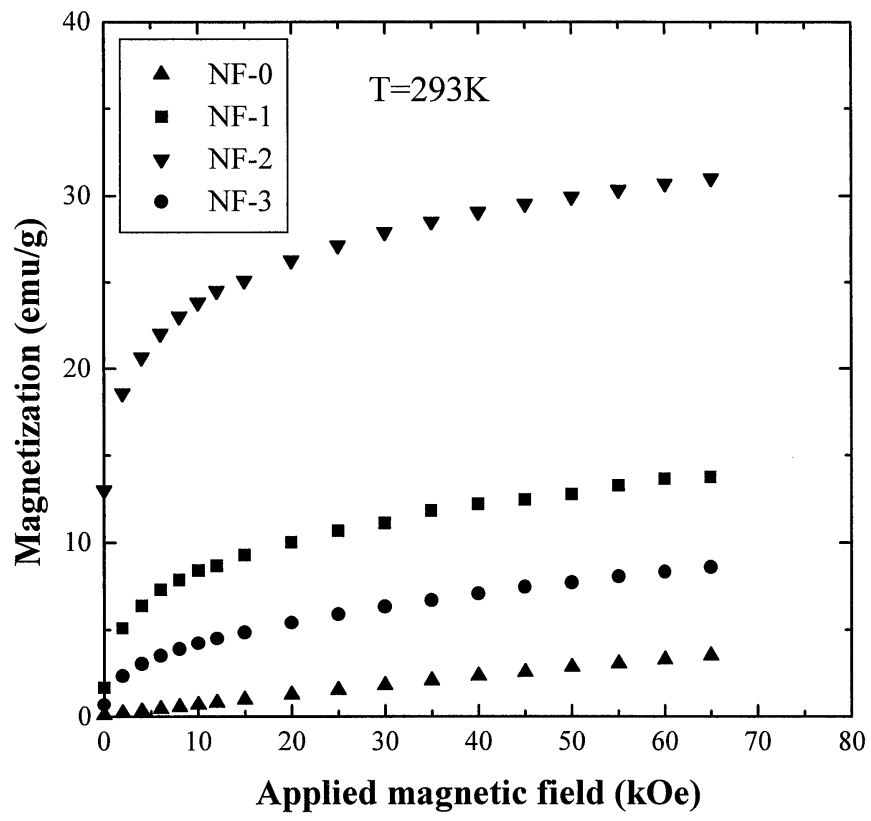

Fig. 3. M-H curves of nickel ferrite synthesized by different methods and the precursor (The notations of samples are the same as in Fig. 1).

The above-mentioned results indicate that the nanosized nickel ferrite was obtained by shock wave treatment of the nanosized ferric oxide and nickel oxide precursor. The main reason for the formation of nanosized nickel ferrite may be that the acting period of shock wave is very short, so the crystallites of nickel ferrite formed do not have enough time to grow to large and perfect crystallites. The nanosized nickel ferrites with different sizes were obtained from varying packing densities of the samples at the same velocity

Table 1

Distances between lattice planes of nickel ferrite samples obtained by electron diffraction patterns

\begin{tabular}{|c|c|c|c|c|c|c|}
\hline \multirow{2}{*}{$\begin{array}{l}\text { Samples } \\
\text { NF-3 }\end{array}$} & \multirow{2}{*}{$\begin{array}{l}\text { Method of } \\
\text { preparation }\end{array}$} & \multicolumn{5}{|c|}{ Distance between lattice planes (nm) } \\
\hline & & 0.293 & 0.250 & 0.206 & 0.159 & 0.147 \\
\hline NF-1 & $\begin{array}{l}\text { Shock wave } \\
\text { treatment }\end{array}$ & 0.291 & 0.248 & 0.205 & 0.158 & 0.146 \\
\hline $\begin{array}{c}\text { Standard } \\
\text { nickel } \\
\text { ferrite* }\end{array}$ & ----- & 0.2948 & 0.2513 & 0.2085 & 0.1605 & 0.1476 \\
\hline $\begin{array}{l}\text { (hkl) index of } \\
\text { lattice } \\
\text { planes }\end{array}$ & ------ & $(220)$ & $(311)$ & $(400)$ & $(511)$ & $(440)$ \\
\hline
\end{tabular}

\footnotetext{
* taken from [9].
} 
of the flying plate, which implies that the particle size of nanomaterials synthesized by shock waves can be controlled by changing the packing density of the samples.

\subsection{Magnetization}

The measured M (Magnetization)-H(Applied magnetic field) curve of the samples before and after shock wave treatment at room temperature is shown in Fig. 3. It can be found that the magnetization of nickel ferrites (NF-1 and NF-2) synthesized by shock wave is obviously higher than that of the samples synthesized by calcination (NF-3). The precursor (NF-0) has the lowest magnetization. The magnetization of NF-2 is higher than that of NF-1, because the crystallinity of NF-2 is better than that of NF-1, which promotes the formation of more perfect magnetic domains that leads to the increase of magnetization. As shown in Fig. 3, the magnetization of the samples NF-2, NF-1, and NF-3 quickly increases as the intensity of applied magnetic field below $30 \mathrm{kOe}$ is increased. The obvious ferrimagnetic behavior of the samples [11] indicates that the ferrimagnetic nickel ferrites were formed by either calcination or shock wave treatment. This is in agreement with the results of XRD and TEM. In succession, the magnetization of the samples NF-2, NF-1, and NF-3 appears to slowly increase linearly with increasing the field above $30 \mathrm{kOe}$ and approaches saturation.

The dependence of the magnetization $\mathbf{M}$ on the applied magnetic field $\mathbf{H}$ can be expressed as,

$$
\mathbf{M}=\chi \mathbf{H}
$$

where $\chi$ is the (constant) susceptibility in terms of Gauss per Oersted (G/Oe). For many ferrimagnetic materials, their susceptibilities $(\chi)$ are greater than $100 \mathrm{G} / O e$. For nickel ferrite $\left(\mathrm{NiFe}_{2} \mathrm{O}_{4}\right)$, its saturated magnetization $\left(\mathrm{M}_{\mathrm{s}}\right)$ is $270 \mathrm{G}$ at $20^{\circ} \mathrm{C}$ [12]. So according to (1), the application of an applied magnetic field of the order of $>2.7 \mathrm{Oe}$, which approximates to that of a ferromagnet [12], may be sufficient to bring the materials practically to saturation. Hence, it is reasonable that the magnetizations of the furnace-reacted nickel ferrite and the two shock-synthesized powders in Fig. 3 would approach saturation at the highest field (65 $\mathrm{kOe})$. Therefore, we can estimate that the saturated magnetizations $\left(\mathrm{M}_{\mathrm{s}}\right)$ of NF-3, NF-1, and $\mathrm{NF}-2$ are 8.6, 13.7, and $31.0 \mathrm{emu} / \mathrm{g}$, respectively.

However, the saturation magnetization of our furnace-reacted nickel ferrite is only $8.6 \mathrm{emu} / \mathrm{g}$, which is far lower than that of the published result (50 emu/g) [12]. We believe that the $M_{s}$ is an intrinsic property of magnetic material, but different preparation methods and conditions have an effect on $\mathrm{M}_{\mathrm{s}}$ of the ferrites in the practical process. Marco et al. [13] have reported that the $\mathrm{M}_{\mathrm{s}}$ of $\mathrm{NiFe}_{2} \mathrm{O}_{4}$ at room temperature is $36 \mathrm{emu} / \mathrm{g}$ by solid-state reaction synthesis, and $19 \mathrm{emu} / \mathrm{g}$ by plasma aerosel fabrication. In this article, the $\mathrm{M}_{\mathrm{s}}$ of our furnace-reacted and shock-synthesized nickel ferrites is generally lower than that of the published result $(50 \mathrm{emu} / \mathrm{g}$ ); this total decrease of the $\mathrm{M}_{\mathrm{s}}$ may be due to an amorphous-like state of the precursor (see Fig.1d). Nevertheless, shock synthesis method still exhibits advantages.

Because shock wave has very high quenching rate (up to $10^{9} \mathrm{~K} / \mathrm{s}$ ) [3], the nonstoichiometric nickel ferrites with high defect and dislocation densities were formed by shock wave treatment in which the distribution of the cations of the sublattice in the spinel is different 
from nickel ferrite synthesized by the traditional calcination. That is, nickel ferrite with higher magnetization than that synthesized by the traditional calcination is synthesized by shock wave treatment.

Based on these preliminary results, it can be concluded that under the action of shock waves nickel oxide and ferric oxide form nanosized nickel ferrite, which has a higher magnetization than that synthesized by the traditional calcination. To have more detailed understanding of the high magnetization of nickel ferrite synthesized by shock wave, further characterizations are needed, which are now in progress in our laboratory.

\section{Conclusions}

The nanosized nickel ferrites with different particle sizes were obtained by shock wave treatment from mixtures of nanosized ferric oxide and nickel oxide precursors with different packing densities. The magnetization of nickel ferrite synthesized by shock wave treatment is significantly higher than that of nickel ferrite synthesized by calcination. The possible reason is that a nonstoichiometric spinel nickel ferrites with a transient structure and a distribution of cations of sublattice different from that of the normal spinel are formed after shock wave treatment due to the very high quenching rate.

\section{Acknowledgments}

This work was supported by the Foundation of Chinese Academy of Engineering Physics, to whom the authors are grateful.

\section{References}

[1] R.A. Graham, B. Morosin, E.L. Venturini, M.J. Carr. Annu. Rev. Mater. Sci. 16 (1986) 15.

[2] N.N. Thadhani, Prog. Mater. Sci. 37 (1993) 117.

[3] T. Sekine, Eur. J. Solid State Inorg. Chem .34 (1997) 823.

[4] R.A. Graham, B. Morosin, Y. Horie, E.L. Venturini, M. Boslough, M. Carr, D.L. Williamson, in: Y.M. Gupta (Ed.), Shock Waves in Condensed Matter-1985, Plenum Press, New York, 1986, p. 693.

[5] B. Morosin, E.L. Venturini, R.A. Graham, D.S. Ginley, Synthetic Metals 33 (1989) 185.

[6] E.L. Venturini, B. Morosin, R.A. Graham, D.L. Williamson, F.L. Williams, Sandia National Laboratories Report No., SAND 87-0315, Los Alamos, New Mexico, 1987

[7] E.L. Venturini, B. Morosin, R.A. Graham, in: S.C. Schmidt, L. Holmes (Eds.), Shock Waves in Condensed Matter-1987, North-Holland, Amsterdam,1988, p. 451.

[8] J.J. Liu, Y.C. Yu, H.L. He, X.G. Jin, K. Xu, Mater. Res. Bull. 35 (2000) 377.

[9] P.D. File, JCPDS File No. 10-325.

[10] E.L. Venturini, B. Morosin, R.A. Graham, Mater. Lett. 3 (1985) 349.

[11] E.L. Venturini, B. Morosin, R.A. Graham, in: Y.M.Gupta (Ed.), Shock Waves in Condensed Matter-1985, Plenum Press, New York, 1986, p. 815.

[12] J. Smit, H.P.J. Wijn, Ferrites, vol. 16, Cleaver-Hume Press, London, 1959, p. 157

[13] M.D. Marco, X.W. Wang, R.L. Snyder, J. Simmins, S. Bayya, M. White, M.J. Naughton, J. Appl. Phys. 73 (1993) 6287. 